\title{
Finite size effects in perturbed boundary conformal field theories
}

\author{
P.Dorey, M.Pillin, A.Pocklington, I.Runkel, R.Tateo, G.M.T.Watts \\ SPhT Saclay, 91191 Gif-sur-Yvette, France, and Dept. Math. Sciences, University of Durham, \\ Durham DH1 3LE, England (PED) \\ ETAS, PTS-A, Borsigstr.10, D-70469 Stuttgart, Germany (MP) \\ IFT/UNESP, Instituto de Fisica Teorica, 01405-900, Sao Paulo - SP, Brasil (AP) \\ King's College London, Strand, London WC2R 2LS, England (IR and GMTW) \\ UVA, Inst. voor Theoretische Fysica, 1018 XE Amsterdam, The Netherlands (RT) \\ E-mail. p.e.dorey@durham.ac.uk, mathias.pilin@etas.de, andrew@ift.unesp.br, \\ ingo@lpthe.jussieu.fr, tateo@ins.uva.nl, gmtw@mth.kcl.ac.uk!
}

ABSTRACT: We discuss the finite-size properties of a simple integrable quantum field theory in $1+1 \mathrm{di}-$ mensions with non-trivial boundary conditions. Novel off-critical identities between cylinder partition functions of models with differing boundary conditions are derived.

\section{Introduction}

A fair amount of work has been devoted in recent years to the investigation of non-perturbative phenomena in integrable quantum field theories. Besides the fact that such systems might be used as a testing ground for general ideas in quantum field theory, such as RG flows and dualities, the wide success of the topic is probably due to its relevance in condensed matter physics. More recently, variants of such systems defined on nontrivial geometries have been considered [i]1]. This is a topic which has an even larger set of potential applications. In condensed matter physics, for example, these include the study of Kondotype systems and the fractional quantum Hall

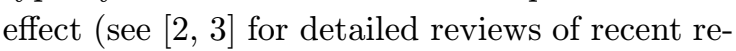
sults in these research areas). String theorists [4] have also shown a certain interest in the subject, and surprisingly connections with other relevant

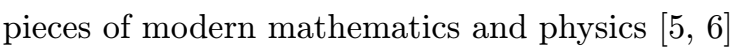
have been discovered.

In this note we shall sketch results more extensively presented in a series of collaborative

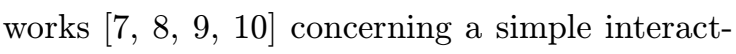
ing quantum field theory confined on a strip-type geometry.
The $\mathcal{M}_{2,5}$ model is perhaps the simplest nontrivial rational Conformal Field Theory (CFT). It has central charge $c=-22 / 5$, and can be identified with the non-trivial $\mathrm{RG}$ fixed point of the $\left(T<T_{c}\right)$ Ising model in a strong purely imaginary magnetic field. This fixed point coincides with the accumulation point of the Lee-Yang zeros and the corresponding conformally invariant theory is known as the Lee-Yang CFT.

The model contains only two irreducible representations of the Virasoro algebra. These have weights 0 and $-1 / 5$, and the corresponding bulk primary fields are the identity $\mathbb{1}$, and a scalar field $\varphi$ of scaling dimension $x_{\varphi}=-2 / 5$. There are two conformally-invariant boundary conditions denoted by $\mathbb{1}$ and $\Phi$ and three relevant boundary fields interpolating pairs of boundary conditions. They all have conformal weight $-1 / 5$. Two of these fields (denoted $\psi$ and $\psi^{\dagger}$ ) interpolate different boundary conditions, while the third $(\phi)$ lives on the $\Phi$ boundary:

$$
\psi \equiv \phi_{-1 / 5}^{(\mathbb{1} \Phi)}, \quad \psi^{\dagger} \equiv \phi_{-1 / 5}^{(\Phi \mathbb{1})}, \quad \phi \equiv \phi_{-1 / 5}^{(\Phi \Phi)} .
$$

\section{The perturbed CFT}

We shall discuss a perturbation of this CFT, the 
scaling Lee-Yang model. On a cylinder of width $R$ and circumference $L$, this has the action

$$
\begin{aligned}
& \mathcal{A}_{B L Y}=\mathcal{A}_{B C F T}+\lambda \int_{0}^{R} \mathrm{~d} x \int_{0}^{L} \mathrm{~d} y \varphi(x, y) \\
& +h_{l} \int_{0}^{L} \mathrm{~d} y \phi(0, y)+h_{r} \int_{0}^{L} \mathrm{~d} y \phi(R, y),
\end{aligned}
$$

with coordinates $0 \leq x \leq R$ across the cylinder, and $0 \leq y<L$ around it. The parameter $\lambda$ determines the bulk mass and we have allowed the possibility of boundary-perturbing fields on the left and right ends of the cylinder, with couplings $h_{l}$ and $h_{r} . \mathcal{A}_{B C F T}$ is the conformally-invariant action on the cylinder with (conformal) boundary conditions $(\Phi, \Phi)$. We shall also consider $(\mathbb{1}, \Phi)$ and $(\mathbb{1}, \mathbb{1})$ boundary conditions which have a similar expression for $\mathcal{A}_{B L Y}$, but lack one or both perturbing boundary fields.

There are two possible Hamiltonian descriptions of the cylinder partition function. In the socalled L-channel representation the rôle of time is taken by $L$ :

$$
\begin{aligned}
Z_{\alpha \beta} & =\operatorname{Tr}_{\mathcal{H}_{(\alpha, \beta)}} e^{-L H_{\alpha \beta}^{\text {strip }}(M, R)} \\
& =\sum_{E_{n} \in \operatorname{spec}\left(H_{\alpha \beta}^{\text {strip }}\right)} e^{-L E_{n}^{\text {strip }}(M, R)},
\end{aligned}
$$

while in the R-channel representation the rôle of time is taken by $R$ :

$$
\begin{aligned}
Z_{\alpha \beta} & =\left\langle\alpha\left|e^{-R H^{\mathrm{circ}}(M, L)}\right| \beta\right\rangle \\
& =\sum_{E_{n} \in \operatorname{spec}\left(H^{\mathrm{circ}}\right)} \frac{\left\langle\alpha \mid \psi_{n}\right\rangle\left\langle\psi_{n} \mid \beta\right\rangle}{\left\langle\psi_{n} \mid \psi_{n}\right\rangle} e^{-R E_{n}^{\operatorname{circ}}(M, L)} .
\end{aligned}
$$

In equation (2.3i) we have used boundary states $|\alpha\rangle,|\beta\rangle \in\{|\mathbb{1}\rangle,|\Phi\rangle\}$ and the eigenbasis $\left\{\left|\psi_{n}\right\rangle\right\}$ of the Hamiltonian $H^{\text {circ }}$, which propagates states living on a circle of circumference $L$. By contrast, $H_{\alpha \beta}^{\text {strip }}$ in (2.2i) propagates states along a strip of width $R$, and acts on the Hilbert space $\mathcal{H}_{(\alpha, \beta)}$ of states on an interval with boundary conditions $\alpha$ and $\beta$ imposed on the two ends.

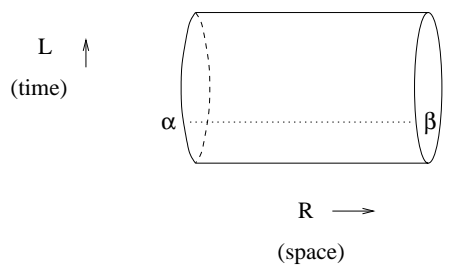

Figure ill: The L-channel decomposition: states $\left|\chi_{n}\right\rangle$ live on the dotted line segment across the cylinder.

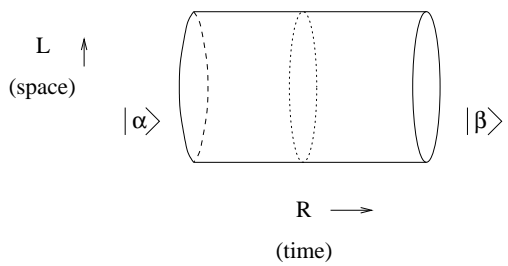

Figure i2: The R-channel decomposition: states $\left|\psi_{n}\right\rangle$ live on the dotted circle around the cylinder.

The two decompositions are illustrated in figures

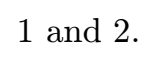

Conformal field theory provides the following useful representations for $H^{\text {circ }}$ and $H^{\text {strip }}$ :

$$
\begin{aligned}
H^{\text {circ }}= & \frac{2 \pi}{L}\left(L_{0}+\bar{L}_{0}-\frac{c}{12}+\lambda\left|\frac{L}{2 \pi}\right|^{\frac{12}{5}} \int_{\theta=0}^{2 \pi} \varphi\left(e^{i \theta}\right) \mathrm{d} \theta\right), \\
H^{\text {strip }}= & \frac{\pi}{R}\left(L_{0}-\frac{c}{24}+\lambda\left|\frac{R}{\pi}\right|^{\frac{12}{5}} \int_{\theta=0}^{\pi} \varphi\left(e^{i \theta}\right) \mathrm{d} \theta\right. \\
& \left.+h_{l}\left|\frac{R}{\pi}\right|^{\frac{6}{5}} \phi_{l}(-1)+h_{r}\left|\frac{R}{\pi}\right|^{\frac{6}{5}} \phi_{r}(1)\right)(2.5)
\end{aligned}
$$

The truncated conformal space approximation, or TCSA, gives numerical estimates for the low-

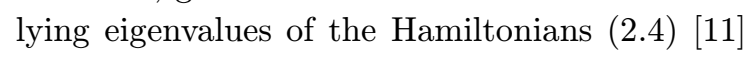

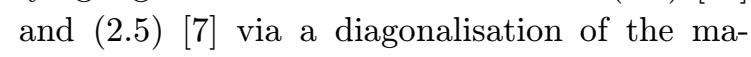
trix $\langle i|H| j\rangle$, where $|i\rangle$ and $|j\rangle$ are states in a finite-dimensional subspace of the relevant CFT Hilbert space.

If the bulk coupling $\lambda$ is set to zero in (2.5), then a purely boundary flow can be studied using the TCSA [i]

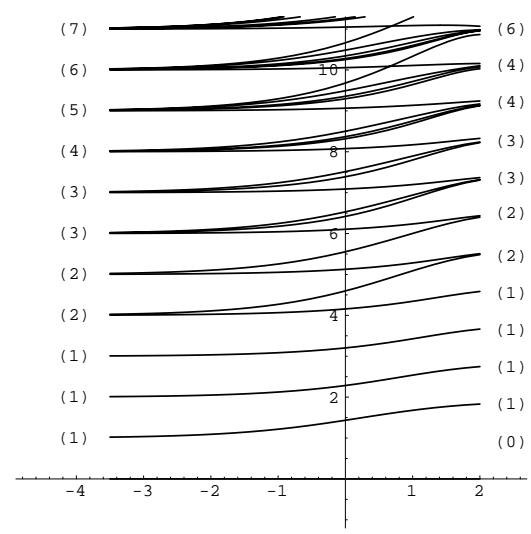

Figure 得: The boundary spectral flow.

The gaps for the excited state scaling functions $F_{n}\left(h^{5 / 6} R\right)=R E_{n}^{\text {strip }}(h, R) / \pi$ are plotted in figure 
on a strip with $(\mathbb{1}, \Phi(h))$ boundary conditions. Note how the multiplicities (written in parentheses) reorganise themselves to give a smooth flow between the conformal $(\mathbb{1}, \Phi)$ and $(\mathbb{1}, \mathbb{1})$ spectra, as encoded in the $\chi_{-1 / 5}$ and $\chi_{0}$ Virasoro characters of $\mathcal{M}_{2,5}$.

In more complicated models the structure of the boundary flows can be much richer; see, for example, Gerard Watts' talk at this conference [1 $12 \pi]$.

\section{The scaling Lee-Yang model}

Turning now to non-zero values of $\lambda$, the scaling Lee-Yang model can also be described by a massive scattering theory consisting of a single selfconjugate particle species $(A=\bar{A})$ with $2 \rightarrow 2$ S-matrix [1] $\left.{ }^{1} \overline{3}_{n}^{\prime}\right]$

$S_{A A}^{A A}(\theta)=S(\theta)=-(1)(2),(x)=\frac{\sinh \left(\frac{\theta}{2}+\frac{i \pi x}{6}\right)}{\sinh \left(\frac{\theta}{2}-\frac{i \pi x}{6}\right)}$.

The physical strip pole at $\theta=2 \pi i / 3$ in $S(\theta)$ corresponds to the on-shell tree diagram involving a non-vanishing " $\varphi$ " coupling represented in figure $\overline{5}$, while the pole at $\theta=i \pi / 3$ describes the same process seen from the crossed channel. The mass $M$ of the particle is related to the bulk perturbation parameter $\lambda$ by $M=\kappa \lambda^{5 / 12}$. The exact value of the constant $\kappa$ was found in [1] 14 in]

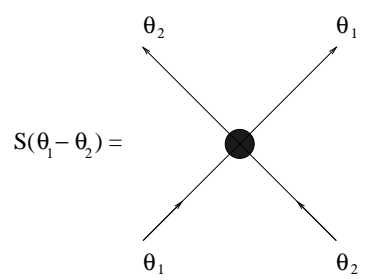

Figure 4 : The the $2 \rightarrow 2$

S-matrix.

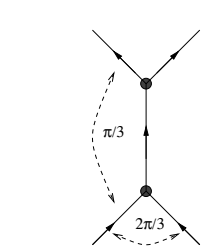

Figure " The " $\varphi^{3}$ " property.
Placing an impenetrable wall at the coordinate position $x=0$, the theory must be supplemented by a reflection factor describing how the particle bounces off the boundary. The reflection amplitudes corresponding to the two integrable bound-

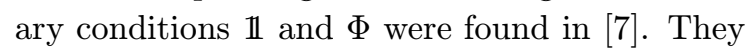
are

$$
R_{\mathbb{1}}(\theta)=\left(\frac{1}{2}\right)\left(\frac{3}{2}\right)\left(\frac{4}{2}\right)^{-1},
$$

and $R_{\Phi(h)}(\theta)=R_{b}(\theta)$, where

$$
R_{b}(\theta)=R_{\mathbb{H}}(\theta)\left(S\left(\theta+i \pi \frac{b+3}{6}\right) S\left(\theta-i \pi \frac{b+3}{6}\right)\right)^{-1}
$$

and

$$
h(b)=-\left|\hat{h}_{\text {crit }}\right| M^{6 / 5} \sin (\pi(b+1 / 2) / 5) .
$$

The exact value of $\hat{h}_{\text {crit }}$ was determined in $9 \overline{9}$. Notice that the reflection factors for the $\mathbb{1}$ and $\Phi(h(0))$ boundaries are identical. However the particle - boundary interaction properties differ: the type $\mathbb{1 1}$ boundary is particle repelling while

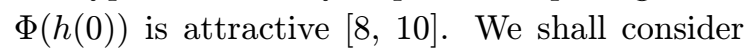
cases where $b$ is real and restrict $b$ to the range $[-3,3]$. The physical poles of $R_{\mathbb{1}}(\theta)$ and $R_{b}(\theta)$ at $\theta=i \pi / 6$ and $\theta=i \pi / 2$ can be explained by postulating a non-vanishing boundary-particle coupling, while the poles at $-i \theta=V_{10}=\pi(b+1) / 6$ and at $-i \theta=V_{20}=\pi(b-1) / 6$ in $R_{b}(\theta)$ lie in the physical strip for $b \in[-1,2]$ and $b \in[1,2]$ respectively. It is natural to associate the latter poles to boundary bound states. The energies $e_{1}$ and $e_{2}$ of these states are given by $e_{j}-$ $e_{0}=M \cos \left(V_{j 0}\right)$, with $e_{0}$ being the energy of the boundary ground-state. Notice that the difference $e_{1}-e_{0}$ is negative for $b \in[2,3]$ and the first boundary bound state becomes, in this range, the true vacuum state. The other poles in the reflection amplitude $R_{b}$ also have field theoretic explanations, but only when a boundary analogue of the Coleman-Thun mechanism is invoked [in]. Further discussion of this aspect of boundary scattering can be found in $\left[1 \overline{1} \overline{5}_{n}^{1}, \overline{1} \overline{6}_{1}\right]$.

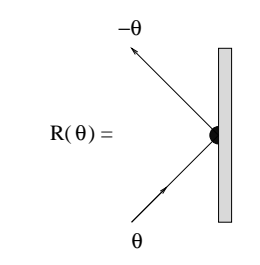

Figure $\overline{6}_{i}^{1}$ The reflection matrix.

\section{The thermodynamic Bethe ansatz}

The Thermodynamic Bethe Ansatz (TBA) [i] $\left.\overline{7}_{1}\right]$ has proved to be a powerful tool in the study of the ground-state energy of integrable quantum field theories on a infinite cylinder. More recently, a variant of the method (the BTBA) 
has been proposed to describe boundary situations [1] equations for the case of the Lee-Yang model was performed. The analysis revealed, for the $(\mathbb{1}, \Phi)$ boundary conditions, that the equations of [1] correctly describe the ground-state energy in the range $b \in[-3,-1]$ but need to be modified for $b \in[-1,2]$ by the inclusion of extra 'active singu-

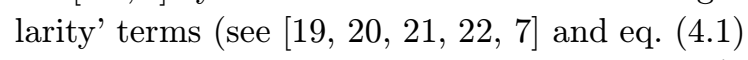
below). Moreover, excited state energies $E_{n}^{\text {strip }}$ can also be computed, using similar generalisations. For the scaling Lee-Yang model the analysis of [1] $1 \overline{1}, \overline{7}]$ led to a non-linear integral equation for a single function $\varepsilon(\theta)$ :

$$
\varepsilon(\theta)=\nu(\theta)+\sum_{p} \log \frac{S\left(\theta-\theta_{p}\right)}{S\left(\theta-\bar{\theta}_{p}\right)}-\mathcal{K} * L(\theta),
$$

plus an associated set of equations for the finite (and possibly empty) set $\left\{\theta_{p}, \bar{\theta}_{p}\right\}$ of 'active' singularities: $\exp \left(\varepsilon\left(\theta_{p}\right)\right)=\exp \left(\varepsilon\left(\bar{\theta}_{p}\right)\right)=-1 \forall p$. In (4.1),$L(\theta)=\log \left(1+e^{-\varepsilon(\theta)}\right)$, the symbol “*” indicates the standard convolution, and

$$
\begin{gathered}
\nu(\theta)=2 R M \cosh \theta-\log \lambda_{\alpha \beta}(\theta), \\
\lambda_{\alpha \beta}(\theta)=R_{\alpha}\left(i \frac{\pi}{2}-\theta\right) R_{\beta}\left(i \frac{\pi}{2}+\theta\right), \\
\mathcal{K}(\theta)=-i \frac{\partial}{\partial \theta} \log S(\theta) .
\end{gathered}
$$

The number of active singularities depends on the particular energy level considered and, as mentioned above, for some pairs of boundary conditions on the strip it is nonzero even for the

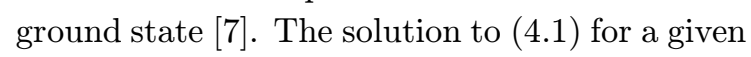
value of $r=R M$ determines a function $c_{n}(r)$ :

$$
\begin{aligned}
c_{n}(r)=\frac{6}{\pi^{2}} \int_{-\infty}^{\infty} d \theta r \cosh \theta L(\theta) \\
\\
\quad+i \frac{12 r}{\pi} \sum_{p}\left(\sinh \theta_{p}-\sinh \bar{\theta}_{p}\right),
\end{aligned}
$$

in terms of which $E_{n}^{\text {strip }}(M, R)=\mathcal{E}_{\text {bulk }} R+f_{\alpha}+$ $f_{\beta}-\frac{\pi}{24 R} c_{n}(r)$, where the $f$ 's are R-independent contributions to the energy from the boundaries and $\mathcal{E}_{\text {bulk }}$ is the bulk energy per unit length. Exact expressions for these quantities can be found, for example, in [1 $10_{0}^{\prime \prime}$. In figure $\overline{1}$ we compare the numerical diagonalisation (TCSA) of $H^{\text {strip }}$ with boundary condition $\left(\mathbb{1},\left.\Phi\right|_{b=-1.5}\right)$ with the numerical solution of the BTBA. No boundary bound states are present. Figure $\overline{9}$ shows instead the situation for ( $\left.\mathbb{1},\left.\Phi\right|_{b=0.8}\right)$ : the first excited state now corresponds to a boundary bound state with an energy gap tending, as $R \rightarrow \infty$, to $\Delta e=e_{1}-e_{0}=\cos \left(\left.V_{10}\right|_{b=0.8}\right)$. Note that the finite-size data has allowed us to confirm the existence of the boundary bound state, inferred at the end of the last section by a completely independent calculation based on the pole structure of the proposed reflection factor.

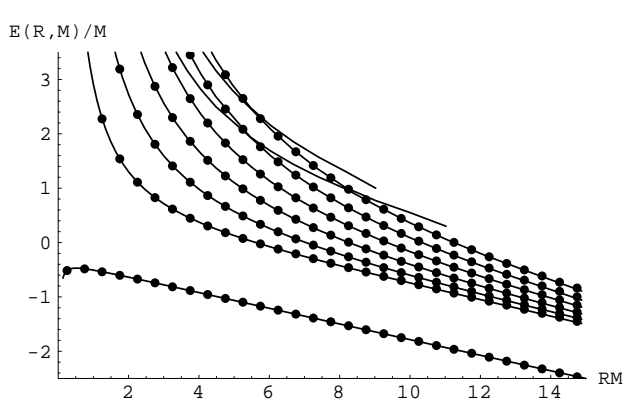

Figure $\overline{1}_{\mathbf{r}}^{\bar{l}}$ TCSA (lines) versus BTBA (dots).

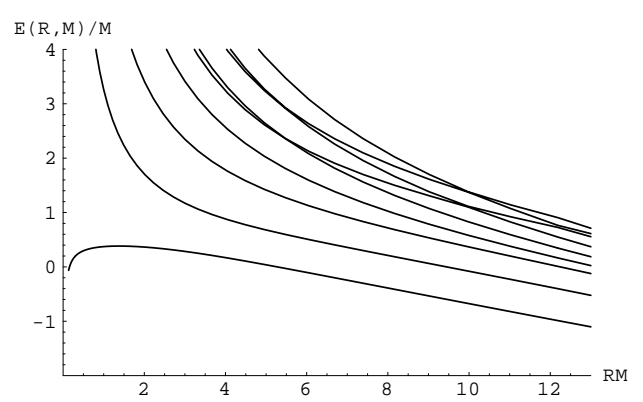

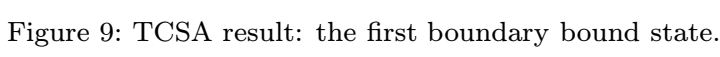

\section{The partition function identities}

Let us now consider the R-channel decomposition of the partition function (2.3i). The follow-

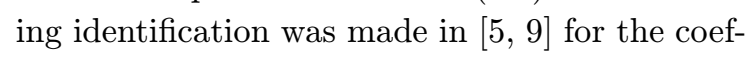
ficients of the weights $\exp \left(-R E_{n}^{\text {circ }}\right)$ :

$$
\left\langle\Phi(h(b)) \mid \psi_{n}\right\rangle=Y_{n}\left(i \pi \frac{b+3}{6}\right)\left\langle\mathbb{1} \mid \psi_{n}\right\rangle,
$$

where $Y_{n}(\theta)=e^{\varepsilon_{n}(\theta)}$, and $\varepsilon_{n}(\theta)$ is the solution of the $n^{\text {th }}$ excited-state TBA equation with periodic boundary conditions. These equations are simply recovered by setting $\nu(\theta)=L M \cosh \theta$ and $r=$

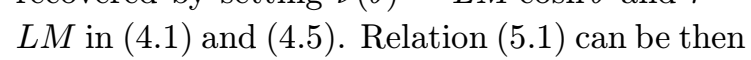


used to write the partition function as

$$
\begin{aligned}
& Z_{\Phi\left(h\left(b_{r}\right)\right), \Phi\left(h\left(b_{l}\right)\right)}=\sum_{n}\left(\frac{\left\langle\mathbb{1} \mid \psi_{n}\right\rangle\left\langle\psi_{n} \mid \mathbb{H}\right\rangle}{\left\langle\psi_{n} \mid \psi_{n}\right\rangle}\right. \\
& \left.\quad Y_{n}\left(i \pi \frac{b_{r}+3}{6}\right) Y_{n}\left(i \pi \frac{b_{l}+3}{6}\right) e^{-R E_{n}^{\text {circ }}(M, L)}\right) .
\end{aligned}
$$

Recalling that the Y's satisfy the functional relation [2] $\left.{ }^{2}\right]^{1}$

$$
Y_{n}(\theta+i \pi / 3) Y_{n}(\theta-i \pi / 3)=1+Y_{n}(\theta),
$$

we quickly obtain the following identity

$$
Z_{\Phi(h(-b)), \Phi(h(b))}=Z_{\mathbb{1}, \mathbb{1 1}}+Z_{\mathbb{1}, \Phi(h(2-b))} .
$$

Eq. (5.5i), being valid for arbitrary $R$ and $L$, is equivalent to the following relation between the spectra of models on strips of equal widths but different boundary conditions:

$$
\begin{aligned}
& \left\{E_{n}^{\text {strip }}\right\}_{\Phi(h(-b)), \Phi(h(b))}= \\
& \quad\left\{E_{n}^{\text {strip }}\right\}_{\mathbb{1}, \mathbb{1}} \cup\left\{E_{n}^{\text {strip }}\right\}_{\mathbb{1}, \Phi(h(2-b))} .
\end{aligned}
$$

This rather surprising relationship has been numerically checked using the TCSA approach and it can be considered as a first off-critical extension of identities between conformal partition functions provided in, for example, [24]. We shall now discuss a simple application of (5.4) [i 10.1 .

There are regions in which the model with $\left(\Phi\left(h_{l}\right), \Phi\left(h_{r}\right)\right)$ boundary conditions develops a boundary-induced vacuum instability: an example situation is represented in figure 100 . (The dashes correspond to complex-conjugate pairs of spectral lines.)

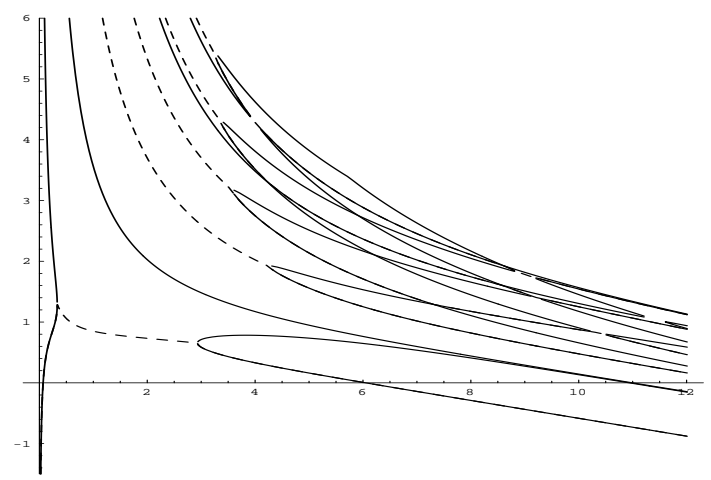

Figure,10' Example of vacuum instability $\left(b_{l}=b_{r}=\frac{1}{2}\right)$. (Axes as on figures si' and 'ig')

${ }^{1}$ Note, the 'ground-state' $Y_{0}$ at $\lambda=0$ coincides with the Stokes multiplier of a Schrödinger equation with $x^{3}$ potential $6 \mid$
These regions are shaded on the $\left(h_{l}, h_{r}\right)$ plane in figure 1111.'. The inner dashed line delimits the values of $h_{l}$ and $h_{r}$ covered for real values of $b_{l}$ and $b_{r}$, while on the ellipse the identity (5.4) holds. On the ellipse, in the small $R$ region, the energy levels $\left.E_{0}\right|_{\mathbb{1}, \Phi(h(2-b))}$ and $\left.E_{0}\right|_{\mathbb{1}, \mathbb{1}}$ correspond, respectively, to the ground state and to the first excited state in the $(\Phi(h(-b)), \Phi(h(b)))$ model. In the opposite, large- $R$, limit, the energy gap $\Delta E=\left.E_{0}\right|_{\mathbb{1}, \mathbb{1}}-\left.E_{0}\right|_{\mathbb{1}, \Phi(h(2-b))}$ tends for $b>0$ to $f_{\mathbb{1}}-f_{\Phi(h(2-b)}=\sin (b-2) \pi / 6$ and becomes negative for $|b|<2$. This simple fact signals the presence of a level crossing at some intermediate value of $R$. The RHS of ( $(5 . \overline{4})$ prohibits the mixing of the two states and ensures that the crossing will be exact. The set of $b$-values with $\Delta E<0$ corresponds to the portion of the ellipse on figure ii $\overline{1}_{1}^{1}$ which touches the shaded region. Once the line $\Delta b=b_{l}+b_{r}=0$ is left,

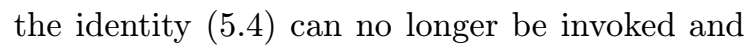
the level crossing it lost. As $\Delta b$ decreases the two levels repel, while in the opposite direction the boundary fields are stronger suggesting the presence of a vacuum instability. Finally in the region $h_{l}<-\left|\hat{h}_{\text {crit }}\right| M^{6 / 5}\left(h_{r}<-\left|\hat{h}_{\text {crit }}\right| M^{6 / 5}\right)$ the reflection factor $R_{b\left(h_{l}\right)}(\theta)$ is not a pure phase for real $\theta$ and, consequently, the vacuum is already unstable in the infinite volume $\bar{i} \overline{7}]$. Shading the region(s) within which, for at least one value of the strip width, the model exhibits a boundaryinduced instability, we end up with the phasediagram represented in figure i1 in. The conjectured scenario has been checked using the TCSA method.

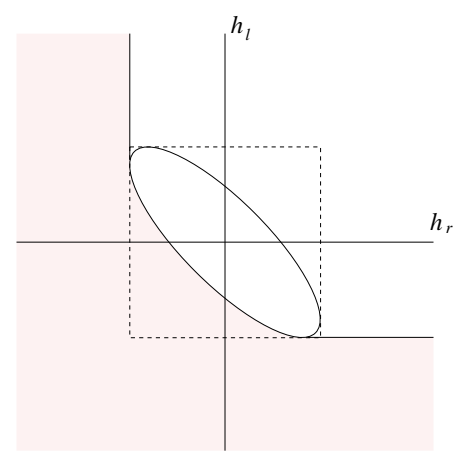

Figure 11 1 ' The phase diagram.

\section{Conclusions}

In this note we have given a brief account of re- 
sults on boundary quantum field theory obtained in the last few years in a series of collaborative papers. Some of the observed phenomena, such as the partition function identities, are expected to be common features of integrable boundary models defined on a cylinder geometry.

Constraints of space and time prevent us from describing two other important topics that can be studied using the example of the boundary scaling Lee-Yang model. In [9], a detailed examination of the flow of the ground-state degeneracy function $g$ was made. It was found that the TBA equations proposed in [1] the variation of $g$ with changes of the bulk mass scale, though if this scale is held fixed, they correctly describe its behaviour as a function of the boundary parameters.

Then the main theme of the recent work [110"] was the computation of correlation functions in a semi-infinite geometry. We refer the interested reader to the original papers for a detailed discussion of these and other issues.

\section{Acknowledgments}

RT thanks Denis Bernard and Bernard Julia for the invitation to speak at this conference. The work was supported in part by a TMR grant of the European Commission, reference ERBFMRXCT960012.

\section{References}

[1] S. Ghoshal and A.B. Zamolodchikov, Int. J. Mod. Phys. A9 (1994) 3841-3886, erratum Int. J. Mod. Phys. A9 (1994) 4353, hep-th/9306002.

[2] H. Saleur, 'Lectures on non perturbative Field Theory and Quantum Impurity Problems: part I', Les Houches Summer School "Topology and Geometry in Physics", July 1998, cond-mat/9812110.

[3] H. Saleur, 'Lectures on non perturbative Field Theory and Quantum Impurity Problems: part II', Nato Advanced Study Institute/EC Summer School on "New Theoretical Approaches to Strongly Correlated Systems", Newton Institute, April 2000, cond-mat/0007309.
[4] J.A. Harvey, D. Kutasov and E.J. Martinec, 'On the relevance of tachyons', hep-th/0003101.

[5] V.V. Bazhanov, S.L. Lukyanov and A.B. Zamolodchikov, Commun. Math. Phys. 177 (1996) 381, hep-th/9412229.

[6] P. Dorey and R. Tateo, J.Phys. A32 (1999) L419, hep-th/9812211 and Nucl. Phys. B563 (1999) 573, hep-th/9906219,

P. Dorey, C. Dunning and R. Tateo, 'Ordinary Differential Equations and Integrable Models', (talk by PED at this conference), PRHEP-tmr2000/034.

[7] P. Dorey, A. Pocklington, R. Tateo and G.M.T. Watts, Nucl. Phys. B525 (1998) 641, hep-th/9712197.

[8] P. Dorey, R. Tateo and G.M.T. Watts, Phys. Lett. B448 (1999) 249, hep-th/9810098.

[9] P. Dorey, I. Runkel, R. Tateo and G.M.T. Watts, Nucl. Phys. B578 (2000) 85, hep-th/9909216.

[10] P. Dorey, M. Pillin, R. Tateo and G.M.T. Watts, 'One-point functions in perturbed boundary conformal field theories', Nucl. Phys. B (to appear), hep-th/0008039.

[11] V.P. Yurov and Al.B. Zamolodchikov, Int. J. Mod. Phys. A5 (1990) 3221.

[12] K. Graham, I. Runkel and G.M.T. Watts, 'Renormalisation group flows of boundary theories', (talk by GMTW at this conference), PRHEP-tmr2000/040, hep-th/0010082.

[13] J.L. Cardy and G. Mussardo, Phys. Lett. B225 (1989) 275.

[14] Al.B. Zamolodchikov, Int. J. Mod. Phys A10 (1995) 1125.

[15] G.W. Delius and G.M. Gandenberger, Nucl. Phys. B554 (1999) 325, hep-th/9904002.

[16] P. Mattsson and P. Dorey, 'Boundary spectrum in the sine-Gordon model with Dirichlet boundary conditions', hep-th/0008071

[17] Al.B. Zamolodchikov, Nucl. Phys. B342 (1990) 695.

[18] A. Leclair, G. Mussardo, H. Saleur and S. Skorik, Nucl. Phys. B453 (1995) 581, hep-th/9503227.

[19] V.V. Bazhanov, S.L. Lukyanov and A.B. Zamolodchikov, Nucl. Phys. B489 (1997) 487, hep-th/9607099. 
[20] P. Dorey and R. Tateo, Nucl. Phys. B482 (1996) 639, hep-th/9607167 and Nucl. Phys. B489 (1998) 575, hep-th/9706140.

[21] D. Fioravanti, A. Mariottini, E. Quattrini and F. Ravanini, Phys. Lett. B390 (1997) 243, hep-th/9608091.

[22] P. Fendley, Adv.Theor.Math.Phys.1 (1998) 210 , hep-th/9706161.

[23] Al.B. Zamolodchikov, Phys. Lett. B253 (1991) 391.

[24] R.E. Behrend, P.A. Pearce and J.-B. Zuber, J. Phys. A31 (1998) L763, hep-th/9807142. 